\title{
Front Matter: Volume 10410
}

, "Front Matter: Volume 10410," Proc. SPIE 10410, Unconventional and Indirect Imaging, Image Reconstruction, and Wavefront Sensing 2017, 1041001 (14 November 2017); doi: 10.1117/12.2296018

SPIE Event: SPIE Optical Engineering + Applications, 2017, San Diego, California, SPIE. United States 


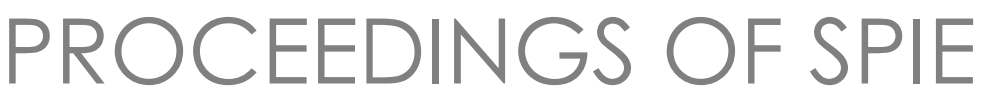

\section{Unconventional and Indirect Imaging, Image Reconstruction, and Wavefront Sensing 2017}

Jean J. Dolne

Rick P. Millane

Editors

9-10 August 2017

San Diego, California, United States

Sponsored and Published by

SPIE 
The papers in this volume were part of the technical conference cited on the cover and title page. Papers were selected and subject to review by the editors and conference program committee. Some conference presentations may not be available for publication. Additional papers and presentation recordings may be available online in the SPIE Digital Library at SPIEDigitalLibrary.org.

The papers reflect the work and thoughts of the authors and are published herein as submitted. The publisher is not responsible for the validity of the information or for any outcomes resulting from reliance thereon.

Please use the following format to cite material from these proceedings:

Author(s), "Title of Paper," in Unconventional and Indirect Imaging, Image Reconstruction, and Wavefront Sensing 2017, edited by Jean J. Dolne, Rick P. Millane, Proceedings of SPIE Vol. 10410 (SPIE, Bellingham, WA, 2017) Seven-digit Article CID Number.

ISSN: 0277-786X

ISSN: 1996-756X (electronic)

ISBN: 9781510612778

ISBN: 9781510612785 (electronic)

Published by

SPIE

P.O. Box 10, Bellingham, Washington 98227-0010 USA

Telephone +1 3606763290 (Pacific Time) · Fax +1 3606471445

SPIE.org

Copyright (C) 2017, Society of Photo-Optical Instrumentation Engineers.

Copying of material in this book for internal or personal use, or for the internal or personal use of specific clients, beyond the fair use provisions granted by the U.S. Copyright Law is authorized by SPIE subject to payment of copying fees. The Transactional Reporting Service base fee for this volume is $\$ 18.00$ per article (or portion thereof), which should be paid directly to the Copyright Clearance Center (CCC), 222 Rosewood Drive, Danvers, MA 01923. Payment may also be made electronically through CCC Online at copyright.com. Other copying for republication, resale, advertising or promotion, or any form of systematic or multiple reproduction of any material in this book is prohibited except with permission in writing from the publisher. The CCC fee code is 0277$786 \mathrm{X} / 17 / \$ 18.00$.

Printed in the United States of America.

Publication of record for individual papers is online in the SPIE Digital Library.

\section{SPIE. DIGITAL}

Paper Numbering: Proceedings of SPIE follow an e-First publication model. A unique citation identifier (CID) number is assigned to each article at the time of publication. Utilization of CIDs allows articles to be fully citable as soon as they are published online, and connects the same identifier to all online and print versions of the publication. SPIE uses a seven-digit CID article numbering system structured as follows:

- The first five digits correspond to the SPIE volume number.

- The last two digits indicate publication order within the volume using a Base 36 numbering system employing both numerals and letters. These two-number sets start with $00,01,02,03$, 04, 05, 06, 07, 08, 09, 0A, OB ... 0Z, followed by 10-1Z, 20-2Z, etc. The CID Number appears on each page of the manuscript. 


\title{
Contents
}

\author{
vii Authors \\ ix Conference Committee
}

\section{SESSION 1 WAVEFRONT SENSING AND COMPENSATION}

1041002 Impact of beacon wavelength on phase-compensation performance [10410-1]

1041003 Adaptive optics using a MEMS deformable mirror for a segmented mirror telescope [10410-2]

1041004 Deep-turbulence wavefront sensing using digital holography in the on-axis phase shifting recording geometry [10410-3]

1041005 Shack-Hartmann Electronic Densitometer (SHED) [10410-4]

1041006 Simultaneous measurements of density field and wavefront distortions in high speed flows [10410-5]

SESSION 2 SYNTHETIC APERTURE, SUPER RESOLUTION, HOLOGRAPHIC AND COMPRESSIVE IMAGING, AND TRACKING I

1041009 Improving spatial resolution of the light field microscope with Fourier ptychography [10410-7]

SESSION 3 SYNTHETIC APERTURE, SUPER RESOLUTION, HOLOGRAPHIC AND COMPRESSIVE IMAGING, AND TRACKING II

10410 OB Synthetic-aperture direct-detection coherent imaging (Invited Paper) [10410-9]

$104100 \mathrm{C}$ Method and algorithm for efficient calibration of compressive hyperspectral imaging system based on a liquid crystal retarder [10410-10]

10410 OD Drift in correlation algorithms for high speed video trackers [10410-11]

$10401 \mathrm{OE}$ Spatial-heterodyne sampling requirements in the off-axis pupil plane recording geometry for deep-turbulence wavefront sensing [10410-12]

10410 OF Local sharpening and subspace wavefront correction with predictive dynamic digital holography [10410-50] 
$104100 \mathrm{U}$ Use of (N-1)-D expansions for N-D phase unwrapping in MRI [10410-13]

$10410 \mathrm{OH} \quad$ High resolution depth reconstruction from monocular images and sparse point clouds using deep convolutional neural network [10410-14]

104100 Improving 3D registration by upsampling of sparse point cloud through fusion with highresolution 2D image [10410-16]

10410 0J Rapid 3D registration using local subtree caching in iterative closest point (ICP) algorithm [10410-17]

\section{SESSION 5 ADAPTIVE OPTICAL SYSTEM, PHASE RETRIEVAL, GHOST AND SHADOW IMAGING}

10410 OK Phase retrieval in the presence of multiplicative noise (Invited Paper) [10410-19]

$10410 \mathrm{OL}$ Fast adaptive optical system for the high-power laser beam correction in atmosphere [10410-20]

$104100 \mathrm{M}$ Laser beam focusing through the atmosphere aerosol [10410-21]

10410 ON Phase retrieval for crystalline specimens [10410-22]

1041000 Resolution limits for shadow imaging of geosynchronous satellites: analytic and simulated approaches [10410-24]

\section{SESSION 6 STRUCTURED ILLUMINATION, SPECKLE, AND UNDERWATER IMAGING}

10410 OP Opportunities for sub-wavelength imaging based on motion in structured illumination (Invited Paper) [10410-25]

$104100 Q \quad$ Long-range speckle imaging theory, simulation, and brassboard results [10410-26]

10410 OR An underwater turbulence degraded image restoration algorithm [10410-27]

10410 OS Object detection from images obtained through underwater turbulence medium [10410-28]

SESSION 7 IMAGE SIMULATION AND PERFORMANCE IMPROVEMENT TECHNIQUES: HARDWARE AND SOFTWARE

10410 0W Unconventional imaging with contained granular media [10410-32]

10410 0X Modeling coherence propagation in a homogenizing light pipe for speckle mitigation [10410-33] 
10410 OY Angle-of-arrival variance of waves and rays in strong atmospheric scattering: split-step simulation results [10410-34]

\section{POSTER SESSION}

1041010 An edge sensitive 3D measurement using two directional laser stripes scanning with a laser projector [10410-18]

1041012 Imaging processing with bidirectional modulation in down-looking synthetic aperture imaging ladar [10410-37]

1041013 Influence of polarization characteristic of targets on synthetic aperture imaging ladar [10410-38]

1041014 A three dimensional point cloud registration method based on rotation matrix eigenvalue [10410-39]

1041015 A multi-resolution texture fusion algorithm [10410-40]

1041016 A novel 360-degree shape measurement using a simple setup with two mirrors and a laser MEMS scanner [10410-41]

1041018 Super-resolution imaging with one complex filter based on compressive sensing [10410-43]

1041019 Optical method of separating of isotropic and anisotropic parts of polymer-dispersed liquid crystals images [10410-44]

$104101 \mathrm{~A}$ Adaptive compressed photon counting 3D imaging based on wavelet trees and Hadamard multiplexing [10410-45]

10410 1B Using virtual reality to test the regularity priors used by the human visual system [10410-46]

10410 1D A Wiener filter approach to shape from shading [10410-48] 
Proc. of SPIE Vol. $104101041001-6$

Downloaded From: https://www.spiedigitallibrary.org/conference-proceedings-of-spie on 26 Apr 2023 Terms of Use: https://www.spiedigitallibrary.org/terms-of-use 


\section{Authors}

Numbers in the index correspond to the last two digits of the seven-digit citation identifier (CID) article numbering system used in Proceedings of SPIE. The first five digits reflect the volume number. Base 36 numbering is employed for the last two digits and indicates the order of articles within the volume. Numbers start with $00,01,02,03,04,05,06,07,08,09,0 A, 0 B . .0 Z$, followed by 10-1Z, 20-2Z, etc.

\begin{tabular}{|c|c|}
\hline Alexandrov, Alexander, OL & Liu, Liren, 12, 13 \\
\hline Arnal, Romain A., ON & Lu, Wei, 13 \\
\hline August, Isaac, OC & Lu, Zhiyong, 12, 13 \\
\hline Banet, Matthias T., OE & Luan, Zhu, 12 \\
\hline Basinger, Scott, ow & Luo, Qiaoen, OP \\
\hline Bones, Philip J., OG & Lylova, Anna, OL \\
\hline Brennan, Terry J., 02 & Maksymyak, Peter P., 19 \\
\hline Buckner, Benjamin, 06 & Mao, Tianyi, lA \\
\hline Burrell, Derek J., 02 & Millane, Rick P., OG, ON \\
\hline Castillo, Oscar E., ID & Miura, Kenjiro T., 09 \\
\hline Chen, Joe P. J., OK & Miyamura, Norihide, 03 \\
\hline Chen, Qian, 1A & Moore, Trevor D., OX \\
\hline Chen, Yulu, OP & Muñoz, Antonio, 1D \\
\hline Dai, Huidong, lA & Muschinski, Andreas, oY \\
\hline Dayton, David C., OD & Nehrych, Andriy L., 19 \\
\hline Dimitrievski, Martin, $\mathrm{OH}$ & Nikitin, Alexander, OM \\
\hline Dolne, Jean, Ol, OJ & Nolasco, Rudolph, OD \\
\hline Douglas, Dennis M., 00 & Oiknine, Yaniv, OC \\
\hline Enterline, Allison A., 02 & Palmer, Eric, 1B \\
\hline Fei, Zi Xuan, 14, 15 & Perram, Glen P., 04 \\
\hline Fienup, James R., OB & Philips, Wilfried, $\mathrm{OH}$ \\
\hline Flores, Jorge L., ID & Pizlo, Zygmunt, 1B \\
\hline Furhad, Md. Hasan, OR, OS & Quadrelli, Marco B., OW \\
\hline Galaktionov, llya, OM & Raghuram, Vivek, OP \\
\hline Gao, Xiaofei, 14 & Raynor, Robert A., OX \\
\hline George, Jacob, 06 & Riker, Jim F., OQ \\
\hline Gibson, Steve, OF & Samarkin, Vadim, OL, OM \\
\hline Goossens, Bart, $\mathrm{OH}$ & Schweinsberg, Aaron, 05 \\
\hline Gu, Guohua, 18, 1A & Shecter, Liat, OC \\
\hline Guo, Jia Yu, 10, 15 & Sheldakova, Julia, OL, OM \\
\hline He, Weiji, lA & Sheppard, David G., 00 \\
\hline Hess, Cecil, 06 & Sidick, Erkin, OW \\
\hline Hoffmann, Heiko, 0J & Spencer, Mark F., 02, 04, OE, OX \\
\hline Hou, Peipei, 13 & Stern, Adrian, OC \\
\hline Hunt, Bobby R., 00 & Sui, Xiubao, 18 \\
\hline Jenkins, Thomas, 06 & Sulaiman, Sennan, OF \\
\hline Jin, Rui, 14, 16 & Sun, Jianfeng, 12,13 \\
\hline Kim, Kyungnam, Ol, OJ & Sun, Yicheng, 18 \\
\hline King, Laura J., OG & Tahtali, Murat, OR, OS \\
\hline Kirian, Richard A., OK & Tani, Yoshitake, 09 \\
\hline Kudryashov, Alexis, OL, OM & Thornton, Douglas E., 04 \\
\hline Kwon, Hyukseong, Ol & Trolinger, James, 06 \\
\hline Kwon, TaeKyu, IB & Tyler, Glenn A., OQ \\
\hline Lambert, Andrew, OR, OS & Uhlenbrock, Ryan, OJ \\
\hline Legarda-Saenz, Ricardo, ID & Usuki, Shin, 09 \\
\hline Li, Dong, 10, 16 & Valenzuela, Anthony, 05 \\
\hline Li, Yu Qin, 15 & Vaughn, Jeff L., OQ \\
\hline Li, Yuqi, 18 & Veelaert, Peter, $\mathrm{OH}$ \\
\hline Lin, Dergan, OP & Voelz, David, OY \\
\hline
\end{tabular}


Wang, Chao, 10, 14, 16

Wang, Lijuan, 12, 13

Webb, Kevin J., OP

Wijerathna, Erandi, OY

Xiao, Xifeng, OY

Xu, Changda, 10

$X \cup$, Qian, 12, 13

Yang, Tao, 15, 16

Ye, Ling, $1 \mathrm{~A}$

Zhou, Xiang, 10, 14, 15, 16

Zhou, Yu, 12 


\section{Conference Committee}

Program Track Chairs

Stephen M. Hammel, Space and Naval Warfare Systems Command (United States)

Alexander M. J. van Eijk, TNO Defence, Security and Safety

(Netherlands)

Conference Chairss

Jean J. Dolne, The Boeing Company (United States)

Rick P. Millane, University of Canterbury (New Zealand)

Conference Program Committee

Mark A. Anastasio, Washington University in St. Lovis (United States)

Philip J. Bones, University of Canterbury (New Zealand)

Stephen C. Cain, Air Force Institute of Technology (United States) Joe Chen, Arizona State University (United States)

Richard Clare, University of Canterbury (New Zealand)

David C. Dayton, Applied Technology Associates (United States)

Peter C. Doerschuk, Cornell University (United States)

Veit Elser, Cornell University (United States)

James Fienup, University of Rochester (United States)

Victor L. Gamiz, Air Force Research Laboratory (United States)

Richard B. Holmes, Boeing LTS Inc. (United States)

Kenneth J. Jerkatis, Applied Technology Associates (United States)

Thomas J. Karr, Defense Advanced Research Projects Agency

(United States)

Andrew J. Lambert, UNSW Canberra (Australia)

Liren Liu, Shanghai Institute of Optics and Fine Mechanics (China)

Julian Maclaren, Stanford University (United States)

Sergio R. Restaino, U.S. Naval Research Laboratory (United States)

Mark F. Spencer, Air Force Research Laboratory (United States)

Markus E. Testorf, Dartmouth College (United States)

David G. Voelz, New Mexico State University (United States)

Kevin J. Webb, Purdue University (United States)

David Wojtas, University of Canterbury (New Zealand)

Jong Chul Ye, KAIST (Korea, Republic of)

Chun Hong Yoon, SLAC Stanford University (United States) 


\section{Session Chairs}

1 Wavefront Sensing and Compensation

David C. Dayton, Applied Technology Associates (United States)

2 Synthetic Aperture, Super Resolution, Holographic and Compressive Imaging, and Tracking I

Rick P. Millane, University of Canterbury (New Zealand)

3 Synthetic Aperture, Super Resolution, Holographic and Compressive Imaging, and Tracking II

Rick P. Millane, University of Canterbury (New Zealand)

4 3D Image Reconstruction

James R. Fienup, University of Rochester (United States)

5 Adaptive Optical System, Phase Retrieval, Ghost and Shadow Imaging

Jean J. Dolne, The Boeing Company (United States)

$6 \quad$ Structured Illumination, Speckle, and Underwater Imaging

Mark F. Spencer, Air Force Research Laboratory (United States)

7 Image Simulation and Performance Improvement Techniques: Hardware and Software

Philip J. Bones, University of Canterbury (New Zealand) 